\title{
Algorithm Improvement of the Low-End GNSS/INS Systems for Land Vehicles Navigation
}

\author{
Quan Zhang, Xiaoji Niu, Hongping Zhang, and Chuang Shi \\ GNSS Research Center, Wuhan University, No. 129, Luoyu Road, Wuhan, Hubei 430079, China \\ Correspondence should be addressed to Xiaoji Niu; xjniu@whu.edu.cn
}

Received 29 March 2013; Accepted 8 June 2013

Academic Editor: Yuri Vladimirovich Mikhlin

Copyright ( 2013 Quan Zhang et al. This is an open access article distributed under the Creative Commons Attribution License, which permits unrestricted use, distribution, and reproduction in any medium, provided the original work is properly cited.

\begin{abstract}
Recent advances in MEMS IMUs give the potential to develop affordable low-end GNSS/INS systems for land vehicles navigation (LVN). To improve the performance of low-end GNSS/INS systems, we made detailed quantitative analysis to the computation terms of the INS navigation equation in regard to accuracy impacts and computation loads and then proposed a simplified INS algorithm and adjusted the corresponding Kalman filter of GPS/INS integration. Comprehensive analysis was made to get the quantitative impacts of each simplified term. Results of road test have shown that the degradation of the navigation accuracy caused by the algorithm simplification was much less than that caused by the sensors errors of the MEMS IMU. Meanwhile, the computation load could be reduced by $70 \%$ with the simplified algorithm, and the reduction can go further to reach nearly $95 \%$ by downsampling IMU data rate simultaneously. Therefore, it is feasible to simplify the INS algorithm without losing accuracy and get benefits of reducing the computation load, which can further enhance the real-time performance of the navigation. The work has special significance for the applications that have limited processor resource and request strict real-time response, such as a deeply coupled GPS/INS receiver.
\end{abstract}

\section{Introduction}

Inertial navigation system (INS) is a self-contained navigation technique, which can provide the position, velocity, and attitude of a moving object from a known starting point. However, INS has inadequate accuracy in the long term due to the sensors induced errors, which grow over time, especially MEMS-based INS [1-3]. The global positioning system (GPS) can provide precise position without degradation with time and become to an important technique of land vehicle navigation (LVN) systems. But environmental conditions may cause GPS signal loss or attenuation and degrade the navigation accuracy [4]. GPS/INS integrated systems can compensate each other's weakness and provide more accurate, continuous, and reliable navigation information (including position, velocity, and attitude) compared to the individual GPS or INS for land vehicles. Kalman filtering is popularly used to fuse the navigation information from INS and GPS $[4,5]$.

It is well known that the inertial navigation algorithm plays an important role in the GPS/INS systems. The algorithm must be precise enough to ensure that the INS can improve GPS positioning and provide accurate navigation information during GPS signal loss or attenuation. On the other hand, the algorithm should keep concise to reduce the computation load and ensure the real-time performance, especially for the applications such as vehicle guidance and safety control. However, due to the quick progress of the computer technology, recent researches of the inertial navigation algorithm rarely focused on improving the realtime performance from the algorithm simplification perspective. But the GPS/INS algorithm simplification becomes important today because of the widespread uses of MEMS inertial systems that are of low cost and low grade. Low cost makes the navigation processor have limited MIPS (million instructions per second), and low grade makes the highprecision algorithm not necessary. One example is GPS/INS deeply coupled integration technology. In addition, the high data rate INS computation needs to be inserted into the processor of the receiver, which might be overloaded. The larger the computation load, the higher the power consumption, which is limited for portable devices, including GPS receivers. In a deeply coupled GPS receiver, the satellite signal tracking 
loops will be aided by the motion dynamic information from the INS in a timely manner. Therefore, there are strict MIPS control and real-time requirement to the INS algorithm for some applications of GPS/INS integrated systems.

1.1. MEMS Inertial Sensor. Microelectromechanical systems (MEMS) technology has led to the blooming of low-cost MEMS inertial sensors and MEMS inertial measurement units (IMU). The low-power consumption, light weight, and low cost of these sensors meet the specifications and requirements for many applications, like land vehicle navigation and deeply coupled GPS receiver $[6,7]$. Thus, the evolution in MEMS technology has led to the successful production of low-cost chip-based inertial sensors for measuring angular rates and specific forces. However, due to the inherent limitations of MEMS technology, MEMS IMUs may have large bias instability and noise, which cause the navigation solution to degrade rapidly in the absence of an aiding source, such as GPS, and may be dominant in the navigation errors.

1.2. Previous Works. Inertial navigation algorithm has been studied intensively in the research field of navigation. Savage provided a detailed comprehensive discourse on the analytics of strapdown inertial navigation systems (INS), and the basic technology was used in modern day commercial and military aircraft, guided missiles, surface ships, and underwater vehicles [1]. Titterton and Weston also described the realtime implementation of the inertial navigation algorithm in a computer. And in order to achieve the desired real-time solution of the algorithm, it is proposed that the strapdown computation is split into low, medium, and high frequency sections, and these various computational functions to be implemented are summarized [2].

The INS error analysis can be done with respect to the computer frame or the navigation frame, which will make the corresponding Kalman filter of GPS/INS integration adjustment. Error equations for inertial navigation systems are derived using a perturbation approach (i.e., with respect to the true navigation frame, also called phi-angle approach) and a psi-angle approach (i.e., with respect to the computer frame) [8]. And comparison results have shown that the psi-angle error equation is simpler in form since there is no coupling from the position and velocity errors in the psi misalignment angle equation. Shin also discussed some distinctions in the implementation between the phi-angle approach and a psi-angle approach [9].

Shin proposed a modified INS mechanization equation, which is simple and can be applied to both navigationgrade and lower-grade IMUs. Here, the error dynamics equations were derived based on perturbation analysis. The position was integrated using the second-order Runge-Kutta method, and the quaternion method was used in the attitude integration [10].

Bortz presented an orientation vector mechanization for a strapdown inertial system. The use of this mechanization leads to significant reduction in digital computer time and memory requirements at no sacrifice in accuracy [11].
1.3. Objectives. We will make a comprehensive study on the algorithm improvement of the INS to reduce the computation load and improve the real-time response, in the condition of ensuring the navigation accuracy for low-end vehicle navigation systems. And the algorithm improvements are made based on a standard inertial navigation algorithm. In addition, the corresponding Kalman filter of GPS/INS integration is adjusted accordingly. A typical 15-states loosely coupled KF is chosen as reference for the adjustment. For the analysis of the computation load, the numbers of arithmetic operations are investigated in detail for the standard algorithm and the modified algorithm, including the INS mechanization, the Kalman filtering, and the error states feedback. The reduction percentage of the computation load is given in the end to represent the contribution of the algorithm modification in our paper.

\section{Algorithm Improvement}

Savage and Shin described the standard inertial navigation algorithm in detail. And the dynamic equations for standard inertial navigation algorithm may be expressed as $[1,9]$

$$
\left[\begin{array}{c}
\dot{\mathbf{v}}^{n} \\
\dot{\mathbf{C}}_{n}^{e} \\
\dot{h} \\
\dot{\mathbf{C}}_{b}^{n}
\end{array}\right]=\left[\begin{array}{c}
\mathbf{C}_{b}^{n} \mathbf{f}^{b}-\left(2 \boldsymbol{\omega}_{i e}^{n}+\boldsymbol{\omega}_{e n}^{n}\right) \times \mathbf{v}^{n}+\mathbf{g}_{l}^{n} \\
\mathbf{C}_{n}^{e}\left(\boldsymbol{\omega}_{e n}^{n} \times\right) \\
-\mathbf{v}_{D} \\
\mathbf{C}_{b}^{n}\left(\boldsymbol{\omega}_{i b}^{b} \times\right)-\left(\boldsymbol{\omega}_{i n}^{n} \times\right) \mathbf{C}_{b}^{n}
\end{array}\right] .
$$

All symbols in (1) are defined as follows: $\mathbf{C}_{b}^{n}$ is the rotation matrix from body frame ( $b$-frame, Forward-Right-Down) to navigation frame ( $n$-frame, North-East-Down), $\mathbf{C}_{e}^{n}$ is the rotation matrix from earth frame ( $e$-frame, fixed with respect to the Earth) to $n$-frame representing horizontal position in the algorithm, $\mathbf{f}^{b}$ is the specific force in the $b$-frame (i.e., output of accelerometers), $\mathbf{v}^{n}$ is velocity in the $n$-frame, respectively, $\mathbf{g}_{l}^{n}$ is the normal gravity in the local position in the $n$-frame, $\boldsymbol{\omega}_{i b}^{b}$ is the angular rate of $b$-frame relative to inertial frame ( $i$-frame, nonrotating with respect to the Earth) in the $b$-frame (i.e., output of gyros), $\omega_{n b}^{b}$ is the angular rate of $b$-frame relative to $n$-frame in the $b$-frame, $\omega_{i n}^{n}$ is the angular rate of $n$-frame relative to $i$-frame in the $n$-frame, $\omega_{e n}^{n}$ is the angular rate of $n$-frame relative to $e$-frame in the $n$-frame, $\boldsymbol{\omega}_{i e}^{n}$ is the angular rate of $e$-frame relative to $i$-frame in the $n$ frame, $\boldsymbol{\omega}_{i e}^{e}$ is the angular rate of $e$-frame relative to $i$-frame in the $e$-frame, and $h$ is the ellipsoid height.

In order to reduce the computation load and meet the real-time requirement, the algorithm simplification is carried out. According to the definitions in (1), the dynamic equation is modified as

$$
\left[\begin{array}{c}
\dot{\mathbf{r}}^{n} \\
\dot{\mathbf{v}}^{n} \\
\dot{\mathbf{q}}_{b}^{n}
\end{array}\right]=\left[\begin{array}{c}
D^{-1} \mathbf{v}^{n} \\
\mathbf{C}_{b}^{n} \mathbf{f}^{b}+\mathbf{g}_{l}^{n} \\
0.5 M_{\omega} q_{b}^{n}
\end{array}\right],
$$

where $\mathbf{r}^{n}$ is the position expressed by curvilinear coordinates, $\mathbf{r}^{n}=\left(\begin{array}{lll}\varphi & \lambda & h\end{array}\right)^{T}$ and $\varphi$ is latitude, $\lambda$ is longitude. $D^{-1}$ is the 
transition matrix. $\mathbf{q}_{b}^{n}$ is the quaternion from $b$-frame to $n$ frame, and $M_{\omega}$ is the skew symmetric form of column vector

$$
\begin{aligned}
\boldsymbol{\omega}_{n b}^{b} & =\left(\begin{array}{lll}
\omega_{x} & \omega_{y} & \omega_{z}
\end{array}\right)^{T} \cdot M_{\omega}=M^{\prime}\left(\boldsymbol{\omega}_{n b}^{b}\right) \\
& =\left[\begin{array}{cccc}
0 & -\omega_{x} & -\omega_{y} & -\omega_{z} \\
\omega_{x} & 0 & \omega_{z} & -\omega_{y} \\
\omega_{y} & -\omega_{z} & 0 & \omega_{x} \\
\omega_{z} & \omega_{y} & -\omega_{x} & 0
\end{array}\right], \\
D^{-1} & =\left[\begin{array}{ccc}
\frac{1}{\left(R_{m}+h\right)} & 0 & 0 \\
0 & \frac{1}{\left.\left(R_{n}+h\right) \cos \varphi\right]} & 0 \\
0 & 0 & -1
\end{array}\right],
\end{aligned}
$$

where $R_{m}$ and $R_{n}$ represent the radius of curvature in the meridian and the prime vertical radius of curvature, respectively.

There are some modifications in (2) compared to (1). For instance, the dynamic equation for velocity ignores Coriolis acceleration $\left(2 \boldsymbol{\omega}_{i e}^{n}+\boldsymbol{\omega}_{e n}^{n}\right) \times \mathbf{v}^{n}$, the derivative of position is represented as velocity rather than the rotation rate of $n$ frame relative to $e$-frame, and a quaternion algorithm is used to compute the attitude. Figure 1 summarizes the overall navigation flowchart of the simplified INS algorithm described in this section. Then, the algorithm will be developed based on the modification from (1) to (2).

2.1. Modified Velocity and Position Updates. Refering to (2), the digital integration algorithm for the modified velocity update can be written as

$$
\mathbf{v}_{k}^{n}=\mathbf{v}_{k-1}^{n}+\Delta \mathbf{v}_{f, k}^{n}+\Delta \mathbf{v}_{g, k}^{n}
$$

where $\Delta \mathbf{v}_{g, k}^{n}$ is the velocity increment due to the gravity and $\Delta \mathbf{v}_{f, k}^{n}$ is the velocity increment due to the specific force, which can be written, respectively, as follows:

$$
\begin{gathered}
\Delta \mathbf{v}_{g, k}^{n}=\mathbf{g}_{k-1 / 2}^{n} \Delta t_{k}, \\
\Delta \mathbf{v}_{f, k}^{n}=\frac{1}{2}\left[\mathbf{C}_{n(k-1)}^{n(k)}+I\right] \mathbf{C}_{b(k-1)}^{n(k-1)} \Delta \mathbf{v}_{f, k}^{b(k-1)}, \\
\Delta v_{f, k}^{b(k-1)}=\int_{t_{k-1}}^{t_{k}} C_{b(t)}^{b(k-1)} f^{b} d t \\
\approx \Delta v_{f, k}^{b}+\frac{1}{2} \Delta \theta_{k} \times \Delta v_{f, k}^{b} \\
+\frac{1}{12}\left(\Delta \theta_{k-1} \times \Delta v_{f, k}^{b}+\Delta v_{f, k-1}^{b} \times \Delta \theta_{k}\right) .
\end{gathered}
$$

Here, $\mathbf{C}_{n(k-1)}^{n(k)} \approx I$, which is because the update time is short and the $n$-frame does not basically change from $k-1$ to $k$. The second and third terms on the right-hand side of (6) correspond to the rotational and sculling motions, respectively. Considering that the impact caused by some minor terms to the navigation accuracy may be very small,

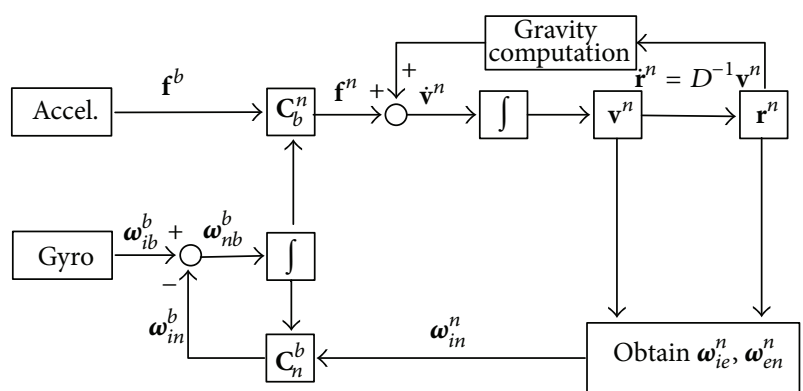

FIGURE 1: The flowchart of modified INS algorithm.

such as rotation correction and sculling correction, then (4) becomes

$$
\mathbf{v}_{k}^{n}=\mathbf{v}_{k-1}^{n}+\mathbf{C}_{b(k-1)}^{n(k-1)} \Delta \mathbf{v}_{f, k}^{b}+\mathbf{g}^{n} \Delta t_{k} .
$$

And then, the second-order Runge-Kutta method is used for the position update when the velocity is known in (2), and the modified position update equation can be written as

$$
\mathbf{r}_{k}^{n}=\mathbf{r}_{k-1}^{n}+D^{-1} \frac{\left(\mathbf{v}_{k}^{n}+\mathbf{v}_{k-1}^{n}\right)}{2} \Delta t
$$

2.2. Modified Attitude Update. For the simplification purpose, angular rate can be assumed as a constant in a short sampling interval $\Delta t$; that is, one-sample algorithm of rotation vector is applied. We can get the analytic discrete solution of attitude quaternion given in (2) since the quaternion algorithm is actually one-sample algorithm. Consider

$$
\mathbf{q}_{k}=\mathbf{q}_{k-1}+\left[\left(\cos \frac{\theta}{2}-1\right) \mathbf{I}+\frac{1}{\theta} \sin \frac{\theta}{2} \mathbf{S}\right] \mathbf{q}_{k-1},
$$

where $\Delta \boldsymbol{\theta}=\Delta \boldsymbol{\theta}_{i b}^{b}-\mathbf{C}_{b}^{n}\left(\boldsymbol{\omega}_{i e}^{n}+\boldsymbol{\omega}_{e n}^{n}\right) \Delta t$, which is the compensated angular increment, and $\theta=|\Delta \boldsymbol{\theta}|, \mathbf{S}=M_{\omega} \Delta t$. Introduce variables

$$
\begin{aligned}
& c=2\left(\cos \frac{\theta}{2}-1\right)=-\frac{\theta^{2}}{4}+\frac{\theta^{4}}{192}+\cdots, \\
& s=\frac{2}{\theta} \sin \frac{\theta}{2}=1-\frac{\theta^{2}}{24}+\frac{\theta^{4}}{1920}+\cdots .
\end{aligned}
$$

Here, taking $c=-\theta^{2} / 4, s=1$; then substitute them into (9) to get modified attitude update equation

$$
\mathbf{q}_{k}=\mathbf{q}_{k-1}+\frac{1}{2}\left(-\frac{\theta^{2}}{4} \mathbf{I}+\mathbf{S}\right) \mathbf{q}_{k-1} .
$$

In summary, the modified digital algorithms for INS are given by

$$
\begin{aligned}
& \mathbf{v}_{k}^{n}=\mathbf{v}_{k-1}^{n}+\mathbf{C}_{b(k-1)}^{n(k-1)} \Delta \mathbf{v}_{f, k}^{b}+\mathbf{g}^{n} \Delta t_{k}, \\
& \mathbf{r}_{k}^{n}=\mathbf{r}_{k-1}^{n}+D^{-1} \frac{\left(\mathbf{v}_{k}^{n}+\mathbf{v}_{k-1}^{n}\right)}{2} \Delta t, \\
& \mathbf{q}_{k}=\mathbf{q}_{k-1}+\frac{1}{2}\left(-\frac{\theta^{2}}{4} \mathbf{I}+\mathbf{S}\right) \mathbf{q}_{k-1} .
\end{aligned}
$$


Additionally, the downsampling method will also be applied because the high data rate might not be necessary for land vehicles and can cause the large amount of computation load. Then, quantitative analysis will be given in order to verify the feasibility of the INS algorithm modifications.

2.3. Adjusted Kalman Filter. Kalman filter (KF) is the most popular estimation technique for GNSS/INS integration. Due to the INS algorithm modification, the corresponding KF should be adjusted, and the corresponding coefficient matrixes in the KF, for example, the state transition matrix, should be simplified because of the truncation of minor error terms of the INS algorithm modification. In addition, a simplified 15-state KF is chosen to be compared to the 21-state $\mathrm{KF}$ in the standard algorithm, where the IMU scale factor errors are excluded. In our paper, the INS error analysis was done with respect to the computer frame (c-frame, locally level at the computed position), which is called the $\psi$-angle error models since the attitude errors are expressed in terms of the $\psi$-angle; for details, see $[8,9]$. Results of quantitative analysis showed that modification on Coriolis acceleration and other minor terms is feasible. Then, the error model of velocity may be written as follows:

$$
\delta \dot{\mathbf{v}}^{c}=\mathbf{f}^{c} \times \psi+\widehat{\mathbf{C}}_{b}^{n} \delta \mathbf{f}^{b} .
$$

Furthermore, the IMU errors are augmented into the KF states and estimated by the difference between GPS and INS solution. The inertial sensor error model is normally built as 1st-order Gaussian-Markov model in most KF implementations for GNSS/INS systems, which can be expressed as [12]

$$
\dot{\mathbf{b}}=-\frac{1}{T} \mathbf{b}+\mathbf{w},
$$

where $\mathbf{b}$ represents the sensors errors and $\mathbf{w}$ is the driving white noise. Compared to the bias error, the effect of the scale factor error is relatively small. Therefore, they are not included in the state vector in our modified algorithm. Combining the simplified navigation error equations based on the $\psi$-angle model and equation (14) to yield the 15-error states equation as follows:

$$
\dot{\mathbf{x}}=F \mathbf{x}+G \mathbf{w},
$$

where $F$ is the dynamic matrix, $G$ is a design matrix, and $\mathbf{x}$ is the state vector as follows:

$$
\mathbf{x}=\left[\begin{array}{lllll}
\delta \mathbf{r}^{c} & \delta \mathbf{v}^{c} & \psi & \mathbf{b}_{g} & \mathbf{b}_{a}
\end{array}\right]^{T},
$$

where $\delta \mathbf{r}^{c}, \delta \mathbf{v}^{c}$ are the position and velocity error vector, $\psi$ is the attitude error, and $\mathbf{b}_{g}$ and $\mathbf{b}_{a}$ are the gyros and accelerometers biases, respectively.

The measurement equation is designed by the loosely coupled closed-loop (feedback) implementation of GNSS/INS integration, as shown in Figure 2. The estimated gyros and accelerometers biases are fed back as compensation to the INS.

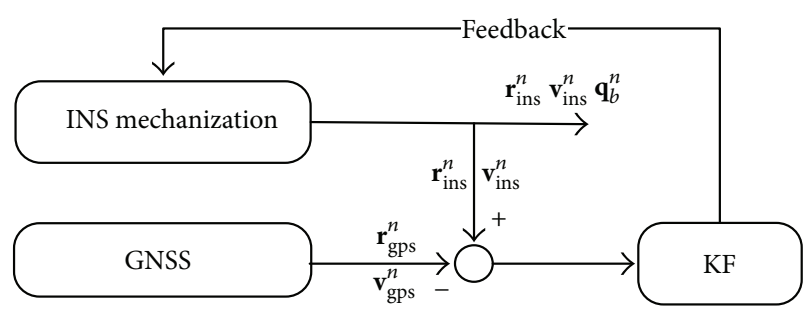

FIGURE 2: Structure of KF for GNSS/INS integration.

\section{Quantitative Analysis to the Modifications}

The proposed modified INS algorithm ignored a number of minor terms, such as rotation and sculling corrections, which are common in the navigation solution. But in order to get its maximum impact on the navigation accuracy, each term should be quantitatively analyzed by assuming its corresponding critical scenarios in the land vehicle situation. Then compare these impacts to the error levels of typical MEMS INS (including both sensor's errors and navigation errors) to decide whether it can be ignored in the low-end navigation systems. So, the following quantitative analysis to the minor terms is conducted under the critical scenarios. For land vehicle navigation, a typical accuracy level of MEMS IMUs is given in Table 1 [13].

3.1. Quantitative Analysis on Coriolis Acceleration. As described, Coriolis acceleration is ignored in (2) compared to (1). In order to analyze the effect of this term, we made the following assumptions on the scenarios of the land vehicles: latitude $40^{\circ}$, velocity $34 \mathrm{~m} / \mathrm{s}$. Table 2 shows the comparison of different acceleration.

From Table 2, it is clear that accelerometer bias is about 10 times greater than the Coriolis acceleration caused by $\boldsymbol{\omega}_{i e}^{n}$, and is approximately 200 times greater than that caused by $\boldsymbol{\omega}_{e n}^{n}$. For low-end GNSS/INS system for land vehicle navigation, the influence of Coriolis acceleration is relatively small and can be ignored.

3.2. Quantitative Analysis on Rotation Correction. In order to illustrate the effect of the rotation motion, consider a situation that a vehicle is maneuvering as in Figure 3 in the lateral plane, that is, simultaneously accelerating laterally and rotating in the yaw plane at a constant speed such that $\mathbf{f}^{b}=$ $\left(\begin{array}{lll}0 & f & -g\end{array}\right)^{T}$ and $\boldsymbol{\omega}^{b}=\left(\begin{array}{lll}0 & 0 & \omega\end{array}\right)^{T}$.

An equivalent acceleration error caused by ignoring the rotation correction may be written as [2]

$$
\delta \mathbf{a}^{n}=\left[\begin{array}{lll}
\frac{f \omega \Delta t}{2}-\cdots & \frac{f \omega^{2} \Delta t^{2}}{6}-\cdots & 0
\end{array}\right]^{T},
$$

where $\delta \mathbf{a}^{n}$ is the acceleration error vector, which is caused by ignoring the rotation correction. Take the case of a vehicle which is accelerating laterally at $0.2 \mathrm{~g}$, whilst traveling at $36 \mathrm{~km} / \mathrm{h}$ as shown in Figure 3 . The associated turn rate is about $0.5 \mathrm{rad} / \mathrm{s}$ (i.e., $28.6 \mathrm{deg} / \mathrm{s}$ ). Assume an update interval of 


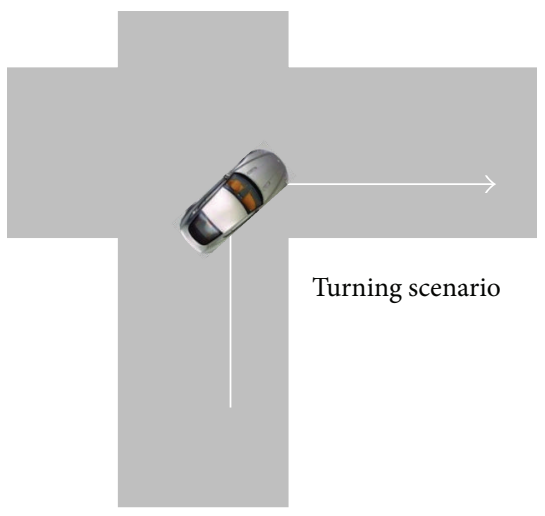

FIgURE 3: Typical scenario of rotation effect of the velocity.

TABle 1: Performance of typical MEMS IMU.

\begin{tabular}{lcc}
\hline & Accel. & Gyro \\
\hline Bias & $3000 \mu \mathrm{g}$ & $36 \mathrm{deg} / \mathrm{h}$ \\
White noise & $2.7 \mathrm{~m} / \mathrm{s} / \mathrm{sqrt}(\mathrm{h})$ & $3.17 \mathrm{deg} / \mathrm{sqrt}(\mathrm{h})$ \\
Position error in 60 s GPS outage & \multicolumn{2}{c}{$50 \mathrm{~m}$} \\
\hline
\end{tabular}

TABLE 2: Comparison of different acceleration (unit: $\mu \mathrm{g}$ ).

\begin{tabular}{lccc}
\hline Axis & Accel. bias & $2 \boldsymbol{\omega}_{i e}^{n} \times \mathbf{v}^{n}$ & $\boldsymbol{\omega}_{e n}^{n} \times \mathbf{v}^{n}$ \\
\hline$X$ & $3 e+3$ & $3.2 e+2$ & $1.5 e+1$ \\
$Y$ & $3 e+3$ & $3.2 e+2$ & $-1.5 e+1$ \\
$Z$ & $3 e+3$ & $3.8 e+2$ & $3.6 e+1$ \\
\hline
\end{tabular}

$0.005 \mathrm{~s}$; then the ignored rotation effect will cause a forward component of acceleration error as follows [2]:

$$
\delta \mathbf{a}_{x}^{n} \approx \frac{f \omega \Delta t}{2}=250 \mu \mathrm{g}
$$

The analysis shows that the rotation correction can be ignored compared to the MEMS accelerometer bias, which is typically $3000 \mu \mathrm{g}$.

3.3. Quantitative Analysis on Sculling Correction. In calculating the change of velocity over a sampling interval, expressed in the body frame, the following correction term may need to be applied [2]:

$$
\delta \mathbf{v}^{b}=\frac{1}{2} \int_{t_{k}}^{t_{k+1}}\left(\Delta \boldsymbol{\theta} \times \mathbf{f}^{b}-\boldsymbol{\omega}^{b} \times \Delta \mathbf{v}\right) d t .
$$

Sculling is characterized by the simultaneous application of in-phase component of angular and linear oscillatory motion with respect to two orthogonal axes [2]. Sculling effect is now analyzed under conditions where the body performs an angular oscillation along the horizontal axis, with simultaneous linear oscillation in the forward direction such that

$$
\Delta \boldsymbol{\theta}=\int_{t_{k}}^{t_{k+1}} \boldsymbol{\omega}^{b} d t, \quad \Delta \mathbf{v}=\int_{t_{k}}^{t_{k+1}} \mathbf{f}^{b} d t
$$

where

$$
\begin{gathered}
\boldsymbol{\omega}^{b}=\left(\begin{array}{lll}
0 & 2 \pi f \theta_{x} \cos 2 \pi f t & 0
\end{array}\right)^{T}, \\
\mathbf{f}^{b}=\left(\begin{array}{lll}
A_{f} \sin (2 \pi f t+\phi) & 0 & 0
\end{array}\right)^{T},
\end{gathered}
$$

in which $\theta_{x}$ is the angular amplitude of the motion and $A_{f}$ is the amplitude of the cyclic acceleration.

Over the time interval $\Delta t$, an acceleration error arises if sculling correction term is not applied. This error may be expressed as

$$
\delta \mathbf{a}^{b}=\frac{1}{2} \theta_{x} A_{f} \cos \phi\left(1-\frac{\sin 2 \pi f \Delta t}{2 \pi f \Delta t}\right) .
$$

According to the IMU signals in the real road tests, there exist linear vibrations and angular vibrations simultaneously. Consider a situation in which the vehicle exhibits motion at a frequency $f=20 \mathrm{~Hz} . \theta_{x}$ is taken to be $0.02 \mathrm{deg}$. The cyclic acceleration, with respect to an orthogonal axis, is taken to vary between $\pm A_{f}$, where $A_{f}=0.5 \mathrm{~g}$. According to (22), $b_{a}$ is maximized when $\phi=0$. Under such conditions, the motion of the vehicle is referred to as sculling. Assume an update interval of $0.005 \mathrm{~s}$; then the magnitude of the acceleration error is about $5.6 \mu \mathrm{g}$ derived from (22), which is about 1/536 times smaller than the MEMS accelerometer bias $(3000 \mu \mathrm{g})$. It is clear that the sculling correction term, given in (6), can be ignored.

3.4. Quantitative Analysis on Coning Correction. Coning refers to the motion which arises when a single axis of a body describes a cone, or some approximation to a cone, in space. Such motion results from the simultaneous application of angular oscillations about two orthogonal axes of the system where the oscillations are out of phase [2].

It is assumed here that the body has angular oscillations at a frequency $f$ around the $x$-axis and $y$-axis. The amplitudes of the $x$ and $y$ motions are $\theta_{x}$ and $\theta_{y}$. Additionally, a phase difference of $\phi$ is assumed to exist between the two channels [2]. Thus, we may write

$$
\begin{aligned}
& \boldsymbol{\omega}=2 \pi f\left[\begin{array}{lll}
\theta_{x} \cos 2 \pi f t & \theta_{y} \cos (2 \pi f t+\phi) & 0
\end{array}\right]^{T}, \\
& \boldsymbol{\theta}=\left[\begin{array}{c}
\theta_{x}\left\{\sin 2 \pi f t-\sin 2 \pi f t_{k}\right\} \\
\theta_{y}\left\{\sin (2 \pi f t+\phi)-\sin \left(2 \pi f t_{k}+\phi\right)\right\} \\
0
\end{array}\right] .
\end{aligned}
$$

Coning motion can yield a $z$-component in the computed attitude [2], which can be written as

$$
\delta \theta_{z}=\pi f \theta_{x} \theta_{y} \Delta t \sin \phi\left(1-\frac{\sin 2 \pi f \Delta t}{2 \pi f \Delta t}\right),
$$

where $\Delta t=t_{k+1}-t_{k}$. It can be seen that $\delta \theta_{z}$ is maximized when $\phi=\pi / 2$. Then, the drift error caused by ignoring the coning correction may be expressed as

$$
\delta \dot{\theta}_{z}=\pi f \theta_{x} \theta_{y}\left(1-\frac{\sin 2 \pi f \Delta t}{2 \pi f \Delta t}\right) .
$$


The motion of the body is referred to as coning. Consider a situation where the body exhibits coning motion at a frequency, $f$, of $20 \mathrm{~Hz}$. The angular amplitude of the motion in $x$ and $y$ is taken to be $0.05 \mathrm{deg}$. If the attitude update rate is $200 \mathrm{~Hz}$, the resulting drift derived from (25) is $0.6 \mathrm{deg} / \mathrm{h}$, which is much smaller than the MEMS gyro bias, $36 \mathrm{deg} / \mathrm{h}$. It shows that the coning correction term can be ignored.

3.5. Quantitative Analysis on Data Rate. The previous analysis above shows that the impact the navigation error caused by each ignored term is negligible when the data rate is $100 \mathrm{~Hz}$. Here, different data rates are considered to analyze its impact to the navigation. Since the Coriolis acceleration is not related to the data rate at all, it is not included. Table 3 shows the comparison of impact caused by different data rates.

It can be seen that some terms may not be ignored, such as the rotation correction when the data rate is $10 \mathrm{~Hz}$, because the acceleration error caused by it is slightly larger than the IMU error. But the scenarios (e.g., turning) take only a small portion in the whole navigation mission for the real situation of land vehicle navigation. So, the terms can still be considered to be ignored in the practical implementation, which will be verified by the field-test results in our paper. Meanwhile, the $10 \mathrm{~Hz}$ downsampled data rate is not recommended when the navigation accuracy is the concern.

\section{Analysis on Computation Load}

In order to estimate the relative computer time required by the two different algorithms, the numbers of arithmetic operations, for example, adds (A), subtracts (S), multiplies $(\mathrm{M})$, divides (D), square roots (SR), and trigonometric (T), required were investigated [14]. Here, only those parts of the algorithm that take a large amount of calculations were considered. Concretely, INS mechanization and Kalman filter were investigated in detail. In addition, a large amount of zero elements operations in matrix or vector were omitted when the operations were counted to make the calculations precise. The required numbers of arithmetic operations for these two parts were given in Tables 4 and 5, respectively.

Table 4 presents the number of operations required for INS mechanization for different data rates. From this table, it is clear that the modified algorithm has a better computational efficiency compared to the standard one, and the amount of operations can be reduced by about $72.5 \%$ with the same data rate. On other hand, the data rate also has a great impact on computation load, and the amount of operations can be reduced by $90 \%$, which is proportional to the reduction of the data rate. The computation load can be reduced by about $97 \%$ if both the modified algorithm and the downsampling are applied.

Table 5 presents the numbers of operations required for Kalman filter for one complete cycle, that is, within one second (assuming GPS update of $1 \mathrm{~Hz}$ ). Similar to Table 4, the modified algorithm and the data rate have a great impact on the computation load, which can be reduced by about $69 \%$ and $83 \%$, respectively. Similarly, if the combination of the two factors (the modified algorithm and the data rate) is taken
TABLE 3: Comparison of impacts caused by different data rates.

\begin{tabular}{lccc}
\hline Data rate & $\begin{array}{c}\text { Rotation } \\
\text { effect }(\mu \mathrm{g})\end{array}$ & $\begin{array}{c}\text { Sculling } \\
\text { effect }(\mu \mathrm{g})\end{array}$ & $\begin{array}{c}\text { Coning } \\
\text { effect }(\mathrm{deg} / \mathrm{h})\end{array}$ \\
\hline $200 \mathrm{~Hz}$ & 250 & 5.6 & 0.6 \\
$100 \mathrm{~Hz}$ & 500 & 21 & 2.4 \\
$10 \mathrm{~Hz}$ & 5000 & 87 & 9.8 \\
IMU error & 3000 & 3000 & 36 \\
\hline
\end{tabular}

into account, the numbers of operation can be reduced by nearly $94 \%$, which is a significant improvement.

In summary, the algorithm modification can have a great impact on the computation load, which can be reduced by about $70 \%$. Additionally, Kalman filter also plays a dominant role in the computation load; the adds and subtracts (A\&S) and multiplies (M) are the dominant operations in the total computation load. Furthermore, the data rate has significant impact on the computation load, which reduces the total operation number by approximately $85 \%$ from $100 \mathrm{~Hz}$ to $10 \mathrm{~Hz}$. Clearly, the modified algorithm requires much less computation time than the standard algorithm. If both of the modified algorithm and the downsampling method are taken into account, the computational efficiency can be further improved significantly.

\section{Results and Discussion}

As is well known, the common methods used in evaluating the navigation performance of GPS/INS integration systems is to check the estimation errors of the navigation states when there is stable GPS signals update and estimate the statistic summary of the navigation errors, such as RMS values [6]. However, the methods might not work well when used in evaluating MEMS-based IMU system because the IMU error can be estimated well with a continuous GPS update. The navigation results will drift with time when working in standalone mode, for example, when losing GPS signals. So, their performance should be rated as drift errors versus time. The position drift during GPS signal outages is the most critical indicator of the INS accuracy, and small position drift normally means that other errors of INS (e.g., velocity and attitude error) are also small [15].

Here, we used the methods of simulating GPS outages with different periods during postprocessing and checking the drifts of the navigation states (especially the position drift) during the outages. The INS was assumed to work independently without external aiding sensors (e.g., GPS) for a short-term period (e.g., up to 60 seconds). A set of $60 \mathrm{sec}$ GPS signal outages were simulated during the postprocessing of the field-test datasets to evaluate the overall accuracy of the GPS/INS systems. In addition, the concrete magnitudes of the representative ignored terms in the field test were investigated to see their impacts in detail and to verify the results of the previous quantitative analysis.

To verify the feasibility of the modified algorithm, a representative field test was chosen as an example. The trajectory test was conducted on slightly bumpy roads in the urban area 
TABLE 4: Numbers of operations for different INS algorithms.

\begin{tabular}{|c|c|c|c|c|c|}
\hline & $A \& S$ & M & $\mathrm{D}$ & SR & $\mathrm{T}$ \\
\hline Standard algorithm $(100 \mathrm{~Hz})$ & 32000 & 36600 & 9600 & 200 & 1700 \\
\hline Modified algorithm $(100 \mathrm{~Hz})$ & $8800(27.5 \%)$ & $10400(28 \%)$ & $1600(17 \%)$ & $200(100 \%)$ & $700(41 \%)$ \\
\hline Standard algorithm $(10 \mathrm{~Hz})$ & $3200(10 \%)$ & $3660(10 \%)$ & $960(10 \%)$ & $20(10 \%)$ & $170(10 \%)$ \\
\hline Modified algorithm $(10 \mathrm{~Hz})$ & $880(2.8 \%)$ & $1040(2.8 \%)$ & $160(1.7 \%)$ & $20(10 \%)$ & $70(4 \%)$ \\
\hline
\end{tabular}

Note: adds (A), subtracts (S), multiplies (M), divides (D), square roots (SR), and trigonometric (T).

TABLE 5: Numbers of operations for Kalman filters corresponding to different GPS/INS integration algorithms.

\begin{tabular}{|c|c|c|c|c|c|}
\hline & $A \& S$ & M & $\mathrm{D}$ & SR & $\mathrm{T}$ \\
\hline Standard algorithm $(100 \mathrm{~Hz})$ & 308865 & 403385 & 217 & 107 & 16 \\
\hline Modified algorithm $(100 \mathrm{~Hz})$ & $94610(31 \%)$ & $137707(34 \%)$ & $118(54 \%)$ & $8(7 \%)$ & $4(25 \%)$ \\
\hline Standard algorithm $(10 \mathrm{~Hz})$ & $52095(17 \%)$ & $63095(16 \%)$ & $127(59 \%)$ & $17(16 \%)$ & $16(100 \%)$ \\
\hline Modified algorithm $(10 \mathrm{~Hz})$ & $18200(6 \%)$ & $23407(6 \%)$ & $118(54 \%)$ & $8(7 \%)$ & $4(25 \%)$ \\
\hline
\end{tabular}

Note: adds (A), subtracts (S), multiplies (M), divides (D), square roots (SR), and trigonometric (T).

of the city, which contained various scenarios, as shown in Figure 4. There were 15 different data gaps highlighted by cyan color in Figure 4. Note that several simulated GPS gaps overlap, such as outages \#14 and \#15, which is because the vehicle has been in a stationary state. The moving direction is indicated by the yellow dotted line.

Two different grades of IMUs were used in our paper. The first was SPAN-FSAS tactical grade IMU, which was integrated with a GPS receiver [16]. The second was a typical MEMS INS/GPS system, MTi-G [17]. The mounting of the tested devices was shown in Figure 5. The key specifications of the two IMUs are listed in Table 6.

Results of the tactical grade system (IMU-FSAS) will be presented first to evaluate the degradations introduced by the simplified algorithm quantitatively. Such tactical grade INS is not the objective system in our paper, but we use it to check the absolute degradation that the simplifications can cause. The impacts of the modified algorithm on the navigation accuracy will be shown clearly because the original navigation error of tactical grade system is small. Then, the MEMS system results are given as an example to validate whether the modified algorithm is amenable to MEMS systems.

5.1. Results of Tactical Grade System. Here, the vertical errors are not considered since they are not the major concern in some applications (e.g., land vehicle navigation); so, only horizontal error (position drift error in GPS signal outages) is analyzed in our paper. First, we will analyze the impact of each ignored term on the positioning accuracy with the condition that the data rates are the same.

Figure 6 and Table 7 show the field-test results for the tactical grade system with data rate of $200 \mathrm{~Hz}$, in terms of position drifts in $60 \mathrm{sec}$ GPS gaps. Here, the results of the standard algorithm with the $200 \mathrm{~Hz}$ data rate are taken as the reference.

Figure 6 shows the position drift errors with ignoring each minor term in the INS algorithm, respectively, with the data rate of $200 \mathrm{~Hz}$. It can be seen that the ignored terms have little impact on the position drift error except for the Coriolis acceleration, which has a relatively obvious impact on the position drift error, especially in outages \#3 and \#5. According to the analysis in Section 4, the Coriolis acceleration is mainly related to the velocity and should have a larger impact when traveling with higher speed. This seems to be not the case for the real test results shown in Figure 6. However, it should be noted that the impact caused by ignoring the minor terms can be absorbed into the IMU sensors' errors. When Kalman filter becomes stable, the impact can be well compensated through the online estimated IMU sensors' errors. On the contrary, when the steady state of Kalman filter is broken due to vehicle maneuver, the corresponding sensor error estimation also changes. Therefore, the vehicle maneuver affects the impact of ignoring the minor terms. The statistic summary of the position drift error in simulated GPS outages was listed in Table 7.

From Table 7, it is clear that ignoring the Coriolis acceleration may increase the position drift error by $3.7 \%$, while ignoring other terms has almost no impact on the position accuracy. In this case, the Coriolis acceleration is the main factor of the algorithm simplification. These results are consistent with the theoretical analysis.

Figure 7 and Table 8 show the field-test results when the data rate is reduced to $10 \mathrm{~Hz}$. Here, the results of the standard algorithm with $10 \mathrm{~Hz}$ data rate are taken as the reference.

Figure 7 shows the position drift errors with ignoring each minor term in the INS mechanization, respectively, and the data rate is $10 \mathrm{~Hz}$. It can be seen that the impact of Coriolis acceleration and the rotation correction of velocity cause the major degradations. Moreover, the rotation correction has a more obvious impact on certain outages, such as outages \#5, $\# 11$, and \#13, where the position drift errors were significantly increased without rotation correction. From Figure 4, we can see that there was U-turn or left/right turn motion in these three outages, and the heading change could cause the greater rotational effect as shown in Figure 3. Table 8 is a statistic summary of the position drift error in simulated GPS outages, with $10 \mathrm{~Hz}$ data rate.

Same as Figure 7, Table 8 shows that only the Coriolis acceleration and rotation correction of velocity have some impact on the position drift error. And the rotation 


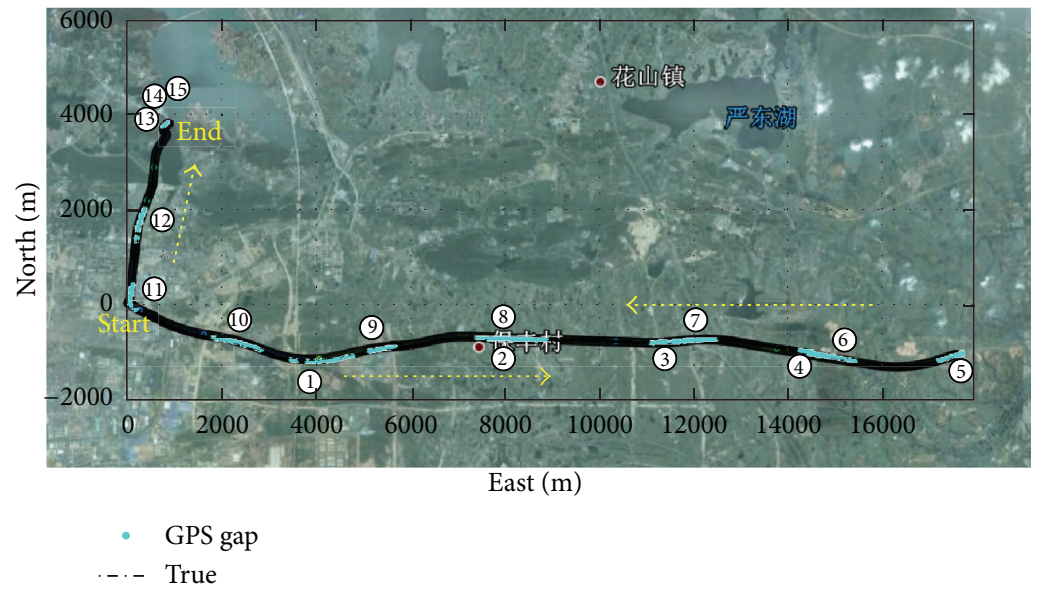

FIGURE 4: Trajectory in urban area and simulated GPS gaps.

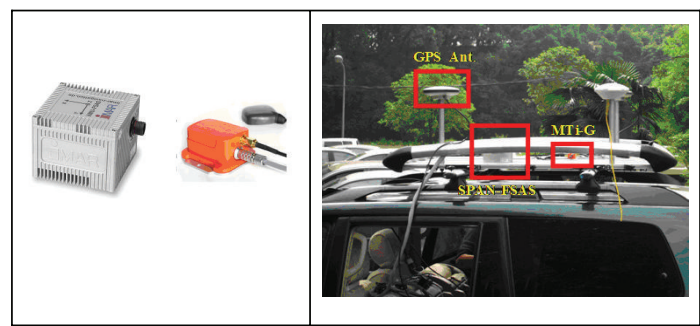

FIGURE 5: Tested IMU sensors (left) and mounting of the tested sensors in test vehicle (right).
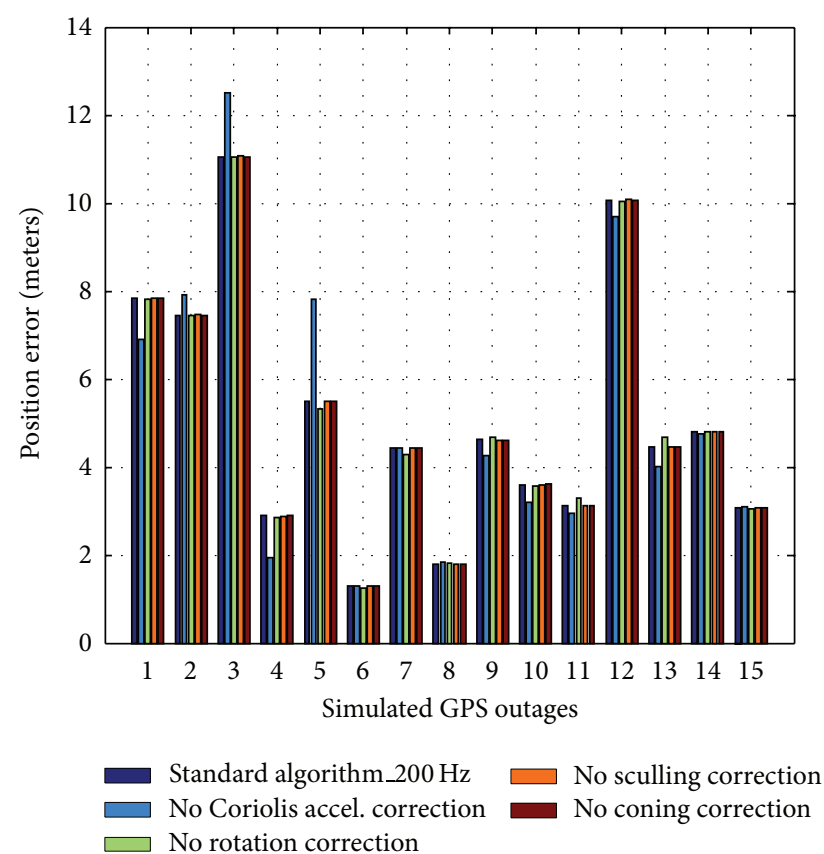

FIGURE 6: Position drift errors in $60 \mathrm{~s}$ GPS outages for different simplifications (tactical grade system, $200 \mathrm{~Hz}$ ).

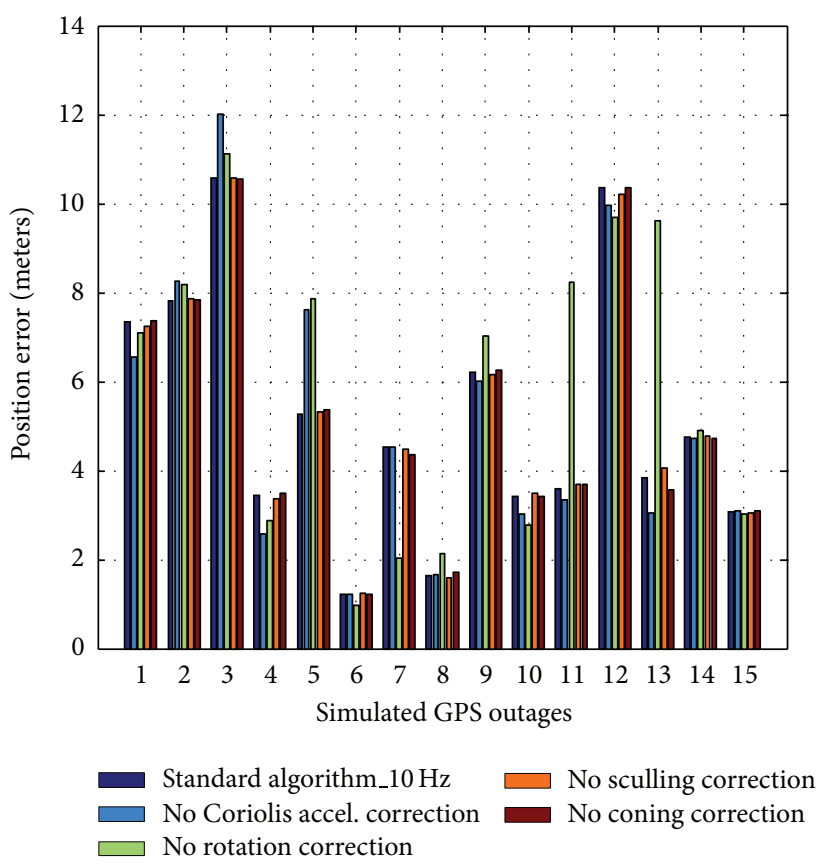

FIgURE 7: Position drift error in $60 \mathrm{~s}$ GPS outages for different simplifications (tactical grade system, $10 \mathrm{~Hz}$ ).

TABLE 6: Specifications of tested IMUs.

\begin{tabular}{lccc}
\hline & & IMU-FSAS & IMU-MTi-G \\
\hline \multirow{2}{*}{ Gyro } & Bias $(\mathrm{deg} / \mathrm{h})$ & 0.75 & 3600 \\
& Noise $(\mathrm{deg} / \mathrm{sqrt}(\mathrm{h}))$ & 0.1 & 3 \\
\hline \multirow{2}{*}{ Accel. } & Bias $(\mu \mathrm{g})$ & 1000 & 2000 \\
& Noise $(\mathrm{m} / \mathrm{s} / \mathrm{sqrt}(\mathrm{h}))$ & 0.03 & 0.12 \\
\hline
\end{tabular}

correction, which affects the position accuracy by about $14.4 \%$, plays an important role in the position drift error. At the same time, the other two terms (e.g., sculling correction 
TABLE 7: Statistic results of position drift errors in $60 \mathrm{sec}$ GPS outages for different simplifications (tactical grade IMU, $200 \mathrm{~Hz}$ ); unit: m.

\begin{tabular}{lccccc}
\hline & Standard algorithm $(200 \mathrm{~Hz})$ & No Coriolis accel. & No rotation correction & No sculling correction & No coning correction \\
\hline Mean & 5.07 & 5.11 & 5.06 & 5.07 & 5.07 \\
RMS & 5.77 & 5.98 & 5.77 & 5.78 & 5.77 \\
Max & 11.05 & 12.52 & 11.06 & 11.06 & 11.05 \\
\hline
\end{tabular}

TABLE 8: Statistic results of position drift errors in 60 sec GPS outages for different simplifications (tactical grade IMU, $10 \mathrm{~Hz}$ ); unit: m.

\begin{tabular}{lccccc}
\hline & Standard algorithm $(10 \mathrm{~Hz})$ & No Coriolis accel. & No rotation correction & No sculling correction & No coning correction \\
\hline Mean & 5.14 & 5.18 & 5.84 & 5.14 & 5.14 \\
RMS & 5.82 & 6.01 & 6.66 & 5.81 & 5.82 \\
Max & 10.57 & 12.00 & 11.13 & 10.57 & 10.57 \\
\hline
\end{tabular}

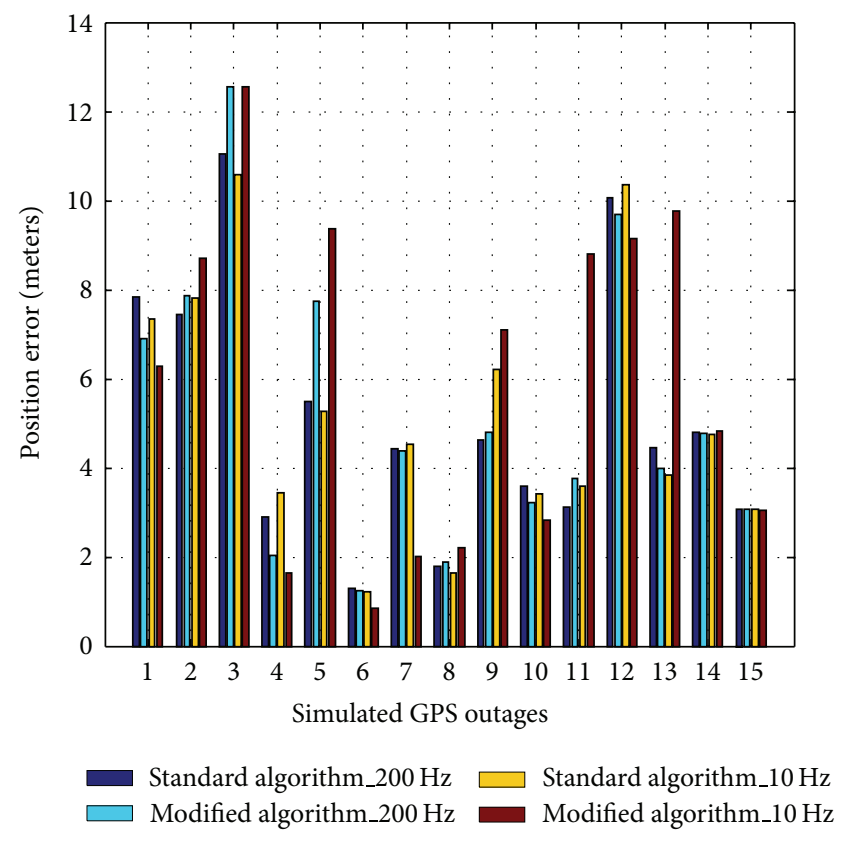

FIGURE 8: Position drift error in 60 s GPS outages with different data rates (tactical grade system, $200 \mathrm{~Hz}$ and $10 \mathrm{~Hz}$ ).

and coning correction) have little impact. This is also consistent with the quantitative analysis in Section 3.

From the previous analysis, it is clear that the impacts on positioning accuracy of each ignored term are related to the data rate except for the Coriolis acceleration. The Coriolis acceleration is the main factor when the data rate is $200 \mathrm{~Hz}$, while the rotation correction is dominant in positioning accuracy when the data rate is $10 \mathrm{~Hz}$. However, the impacts on positioning accuracy of all ignored terms are relatively small in general.

Considering the small impact of each ignored term, we then make an attempt to ignore all the terms simultaneously and analyze the performance as follows. In addition, the data rate is also taken into consideration. Figure 8 shows the comparison of position drift errors caused by the standard algorithm and the modified algorithm when the frequency is $200 \mathrm{~Hz}$ and $10 \mathrm{~Hz}$, respectively.
From Figure 8, it can be seen that the low data rate computation can keep the same positioning accuracy roughly and the position drift error of the modified algorithm degraded more. It is noteworthy that the performance of the modified algorithm was slightly better in some outages such as \#4 and \#7. This may be explained by the stochastic characteristic of the navigation systems. From statistic perspective, such "improvement" should be regarded as the uncertainties of the samples.

In addition, it is obvious that the position drift error appears to be related to the dynamics of the vehicle. When the vehicle undergoes turning or U-turning motion, such as outages $\# 5$, \#11, and \#13, the performance of the modified algorithm becomes worse. On the contrary, when the vehicle moves straight or remains stationary, such as \#2, \#6, and \#14, the impact of the modified algorithm is minimal. The precise comparison was made by the statistic summary in Table 9 .

It can be seen from Table 9 that the low data rate reduces the position accuracy by about $0.9 \%$ with the standard algorithm. And the modified algorithm causes about 4.5\% position degradation over the standard algorithm when the data rate is $200 \mathrm{~Hz}$ and causes about $20 \%$ degradation when the frequency is $10 \mathrm{~Hz}$. The loss of accuracy is not negligible but not significant.

From the results of the field test, we can get the conclusion that the impact of the Coriolis acceleration and the rotation correction are the dominant parts that cause the degradations. Furthermore, downsampling and the modified algorithm reduce the positioning accuracy, but the reduction is relatively small, which is about $20 \%$.

The method of simulating $60 \mathrm{sec}$ GPS outages was applied to demonstrate the impact of the ignored terms, and the results have shown the accuracy loss caused by the algorithm simplification is small. In practice, the land vehicles travel with continuous GNSS signals, that is, navigate in the mode of GNSS and INS integration, most of the time. Therefore, the statistic summary of the navigation errors of the GPS/INS systems with the continuous GPS updates is also given. Table 10 is the RMS of the navigation errors of the tactical grade systems with the continuous GPS updates, including position, velocity, and attitude. From this table, we can see that the algorithm modification has a little impact on 
TABLE 9: Summary of drift error in $60 \mathrm{sec}$ GPS outages (tactical grade IMU); unit: $\mathrm{m}$.

\begin{tabular}{lcccc}
\hline & Standard algorithm $(200 \mathrm{~Hz})$ & Modified algorithm $(200 \mathrm{~Hz})$ & Standard algorithm $(10 \mathrm{~Hz})$ & Modified algorithm $(10 \mathrm{~Hz})$ \\
\hline Mean & 5.07 & 5.19 & 5.14 & 5.94 \\
RMS & 5.77 & 6.03 & 5.82 & 6.93 \\
Max & 11.05 & 12.55 & 10.57 & 12.54 \\
\hline
\end{tabular}

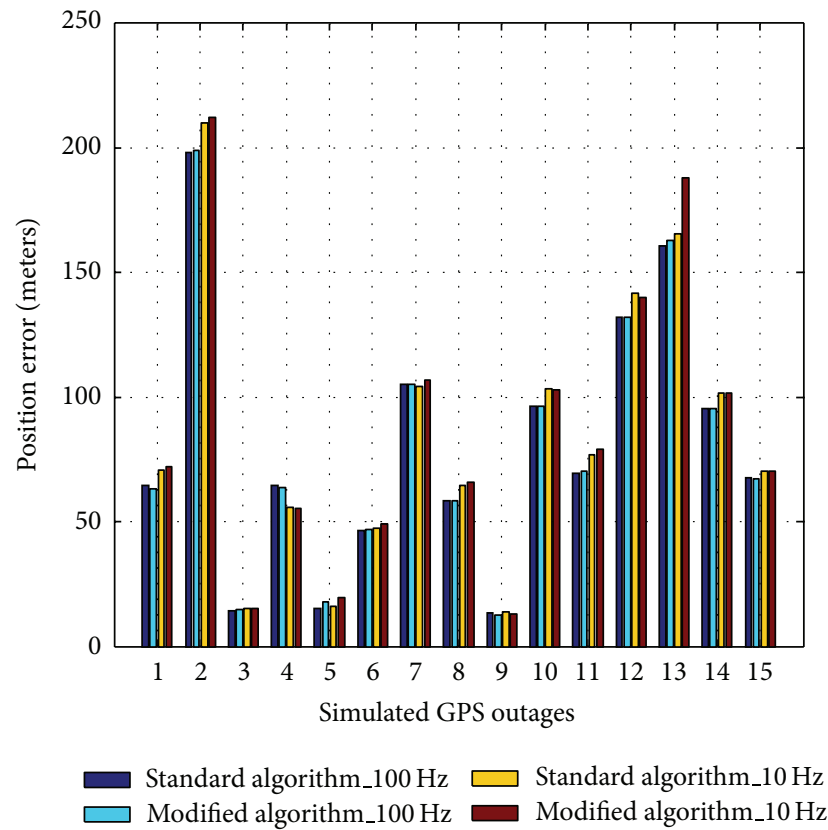

FIGURE 9: Position drift error in $60 \mathrm{~s}$ GPS outages with different algorithms and data rates (MEMS system, $100 \mathrm{~Hz}$ and $10 \mathrm{~Hz}$ ).

the position and velocity accuracy of GPS/INS integration systems, but it has a greater influence on the attitude accuracy, especially the heading. This is because the position accuracy of the integration systems mainly depends on the GPS, while the attitude accuracy mainly depends on the INS, which includes not only IMU performance but also the INS algorithm. However, the impact of the algorithm modification cannot be demonstrated in this case as clear as the methods of simulating GPS outages.

According to the results of the tactical grade systems mentioned earlier, the modified algorithm in fact did not cause much performance degradation. Then, it should make even smaller impact on the lower grade systems, such as MEMS. A field-test dataset of MEMS-based INS/GPS was processed to investigate it.

5.2. Results of MEMS System. Figure 9 and Table 11 show the position drift errors in each GPS outage and statistic summary of the drift error from the MEMS IMU (MTi-G), respectively.

The results from Figure 9 show that, for MEMS systems, the algorithm modification has a small impact on the position drift error, and the low data rate computation can roughly keep the same positioning accuracy as the high data rate computation. The increase of the position drift error is

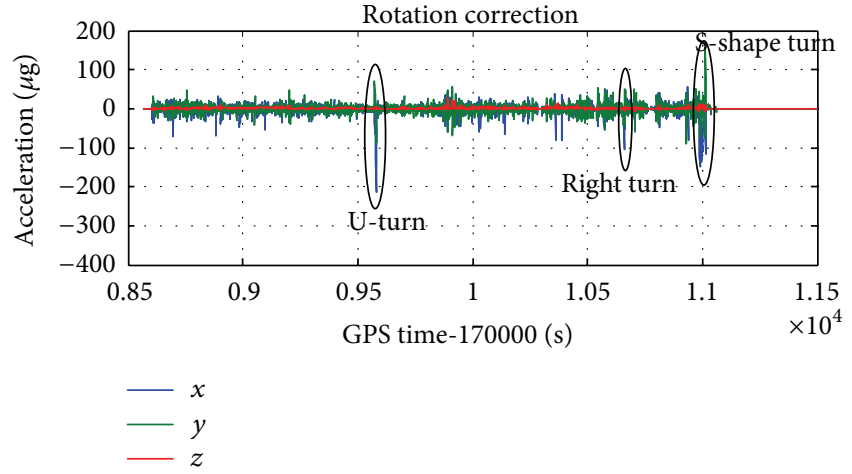

(a)

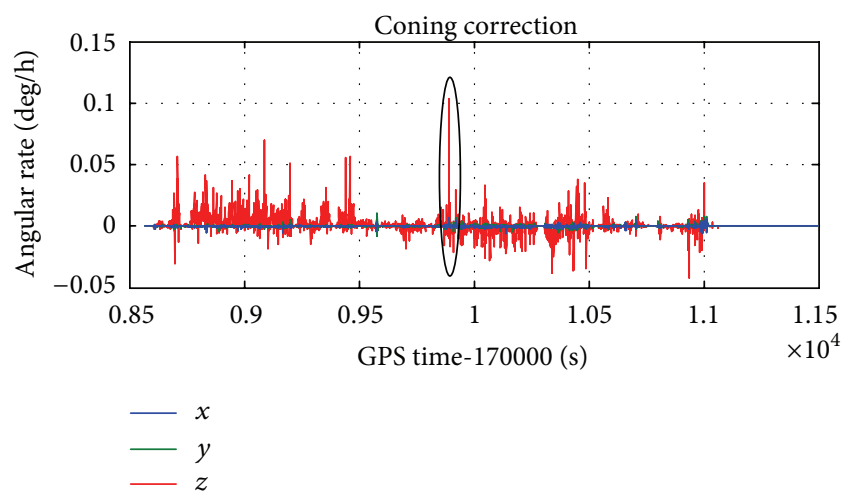

(b)

FIgURE 10: The rotation correction and coning correction in the field test (Tactical grade system).

smaller for the MEMS system compared to that for the tactical grade system.

Statistical results in Table 11 show that the loss of navigation accuracy by the modified algorithm is $0.3 \%$ lower than that by inertial sensor error using the standard algorithm when the calculated frequency is $100 \mathrm{~Hz}$. And the degradation is $8 \%$ with $10 \mathrm{~Hz}$ data rate.

Table 12 gives the statistic summary (RMS) of the navigation errors of the MEMS systems with the continuous GPS updates. Compared to Table 10, the algorithm modifications have little impact on the navigation accuracy for MEMS systems because of the low performance of the MEMS IMU itself.

Results show that the accuracy degradation caused by the modified algorithm is negligible compared to the standard algorithm for MEMS systems. Therefore, the modified algorithm can be applied to the MEMS-based INS/GPS systems for land vehicle navigation. 
TABLE 10: Statistic values (RMS) of navigation errors with continuous GPS updates (tactical grade system).

\begin{tabular}{lcccc}
\hline & Standard algorithm $(200 \mathrm{~Hz})$ & Modified algorithm $(200 \mathrm{~Hz})$ & Standard algorithm $(10 \mathrm{~Hz})$ & Modified algorithm $(10 \mathrm{~Hz})$ \\
\hline Pos_error $(\mathrm{m})$ & 0.059 & 0.057 & 0.059 & 0.055 \\
Vel_error $(\mathrm{m} / \mathrm{s})$ & 0.010 & 0.010 & 0.016 & 0.017 \\
Att_error $(\mathrm{deg})$ & 0.015 & 0.017 & 0.025 & 0.030 \\
Yaw_error $(\mathrm{deg})$ & 0.039 & 0.041 & 0.037 & 0.090 \\
\hline
\end{tabular}

TABLE 11: Summary of drift error in 60 sec GPS outages (MEMS IMU); unit: m.

\begin{tabular}{lcccc}
\hline & Standard algorithm $(100 \mathrm{~Hz})$ & Modified algorithm $(100 \mathrm{~Hz})$ & Standard algorithm $(10 \mathrm{~Hz})$ & Modified algorithm $(10 \mathrm{~Hz})$ \\
\hline Mean & 80.16 & 80.42 & 83.85 & 85.95 \\
RMS & 95.21 & 95.54 & 99.88 & 102.99 \\
Max & 198.20 & 198.93 & 210.26 & 212.09 \\
\hline
\end{tabular}

TABLE 12: Statistic values (RMS) of position errors with continuous GPS updates (MEMS IMU).

\begin{tabular}{lcccc}
\hline & Standard algorithm $(100 \mathrm{~Hz})$ & Modified algorithm $(100 \mathrm{~Hz})$ & Standard algorithm $(10 \mathrm{~Hz})$ & Modified algorithm $(10 \mathrm{~Hz})$ \\
\hline Pos_error $(\mathrm{m})$ & 0.088 & 0.087 & 0.086 & 0.086 \\
Vel_error $(\mathrm{m} / \mathrm{s})$ & 0.033 & 0.032 & 0.034 & 0.033 \\
Att_error $(\mathrm{deg})$ & 0.197 & 0.199 & 0.197 & 0.200 \\
Yaw_error $(\mathrm{deg})$ & 0.672 & 0.670 & 0.675 & 0.674 \\
\hline
\end{tabular}

TABLE 13: Impacts of the modified navigation algorithm for low-end GPS/INS system.

\begin{tabular}{lcc}
\hline & The loss of navigation accuracy & The reduction of computation load \\
\hline Modified algorithm only & $0.3 \%$ & $70 \%$ \\
Downsampling only & $5 \%$ & $85 \%^{*}$ \\
Modified algorithm + downsampling & $8 \%$ & $95 \%$ \\
\hline
\end{tabular}

*: downsample IMU data rate from $100 \mathrm{~Hz}$ to $10 \mathrm{~Hz}$, and consider computation loads of both the INS mechanization and the Kalman filtering.

5.3. Magnitudes of Ignored Terms in Field Test. In addition to the overall performance of the GNSS/INS integrated navigation system, the concrete magnitudes of those ignored terms were investigated using the field-test data. Here, only the impact of rotation and coning was given because the impacts of the two terms are relatively large and typical. A moving-average smoothing method was used in the analysis of the correction results to suppress the interference of sensor noise. Here, the correction results from the tactical grade system (SPAN-FSAS, $200 \mathrm{~Hz}$-sampling) shown on Figure 10 were given to show the impact of the ignored terms better in the field test.

There are three markings indicated by the black circles corresponding to the U-turn, right turn, and S-shape turn, respectively, in the plot of the rotation correction in Figure 10. In the three cases, there is a bigger rotation correction. This is because the turns may create a relative large rotation effect as described in Section 3.3. But the maximum magnitude of the rotation correction in the field test is still smaller than the typical values $(250 \mu \mathrm{g})$ listed in Table 3 . Here, taking the case of U-turn (blue line) as an example, the lateral acceleration is about $0.2 \mathrm{~g}$ with about $0.42 \mathrm{rad} / \mathrm{s}(24 \mathrm{deg} / \mathrm{s})$ of the average rate of the associated turn; then the acceleration correction caused by the rotation effect is only about $210 \mu \mathrm{g}$. In addition, it is clear that the correction of the $z$-axis is small, because the turns rarely happened in the $x-/ y$-axis in vehicle navigation. For the plot of the coning correction in Figure 10, the maximum magnitude of the coning correction, indicated by the black circles, is about $0.1 \mathrm{deg} / \mathrm{h}$, which is also less than the extreme results listed in Table 3. The coning correction along $z$-axis is significantly larger than that of $x$-/ $y$-axis. This is because the angular oscillations along $x$-and $y$-axes of the vehicles are strongly correlated, that is, have the same frequency, which can cause coning effect along $z$-axis. But the angular vibration along $z$-axis has different frequency that of $x-/ y$-axis; therefore, the corresponding coning effect along $x$-/y-axis.

From the previous analysis, it is clear that the quantitative analysis in Section 3 is reasonable, which represent the extreme impacts of the ignored terms. The actual impacts of the ignored terms are small enough in real cases and can be ignored.

\section{Summary and Conclusions}

We have proposed an improved navigation algorithm for lowend GNSS/INS integrated systems, which is composed of a simplified INS algorithm and an adjusted 15-state Kalman filter. Detailed quantitative analysis of impact on the navigation was made for those minor terms (which are considered to 
be neglected) of the INS mechanization, so that readers can refer to it to tailor their algorithm appropriately based on their required accuracy and application scenario. The theoretical analysis and the proposed modified algorithm were verified by the field-test datasets.

The field-test results (accuracy degradation and computation saving) of the improved navigation algorithm for low-end GNSS/INS integrated systems were summarized in Table 13.

Results show that it is feasible to apply the proposed modified inertial navigation algorithm to the low-end land vehicle navigation without sacrificing the navigation accuracy and with the benefit of reducing the computation load significantly. It has special significance for the applications that have strict MIPS limitation and/or request harsh realtime response.

Future research work related to this study may focus on the real-time implementation and application to further test its feasibility and effectiveness. One example is to apply the modified algorithm to the deeply coupled GNSS receiver.

\section{Acknowledgments}

This work was supported in part by the National Natural Science Foundation of China (41174028), the National High Technology Research and Development Program of China (2012AA12A206), the LIESMARS Special Research Fund, the Research Start-up Fund from Wuhan University (618273438), and the Fundamental Research Funds for the Central Universities (201161802020002). Dr. Zhiqin Zhu from the School of Geodesy and Geomatics at Wuhan University is thanked for providing the reference system SPAN-FSAS with relevant cooperation, and Qijin Chen is acknowledged for collecting the field test datasets used in this paper.

\section{References}

[1] P. G. Savage, Strapdown Analytics (part 1 and 2), 2000.

[2] D. H. Titterton and J. L. Weston, Strapdown Inertial Navigation Technology, vol. 17, Peter Peregrinus Ltd., 2004.

[3] C. Jekeli, Inertial Navigation Systems with Geodetic Applications, de Gruyter, 2000.

[4] E. D. Kaplan and C. J. Hegarty, Understanding GPS: Principles and Applications, Artech House Publishers, 2006.

[5] M. S. Grewal and A.P. Andrews, Kalman Filtering: Theory and Practice Using MATLAB, 2008.

[6] X. Niu, S. Nassar, and N. El-Sheimy, "An accurate land-vehicle MEMS IMU/GPS navigation system using 3D auxiliary velocity updates," Navigation, Journal of the Institute of Navigation, vol. 54, no. 3, pp. 177-188, 2007.

[7] X. Niu and N. El-Sheimy, "The development of a low-cost MEMS IMU/GPS navigation system for land vehicles using auxiliary velocity updates in the body frame," in Proceedings of the 18th International Technical Meeting of the Satellite Division of The Institute of Navigation (ION GNSS '05), pp. 2003-2012, Long Beach, Calif, USA, September 2005.

[8] D. O. Benson, "A comparison of two approaches to pureinertial and Doppler-inertial error analysis," IEEE Transactions on Aerospace and Electronic Systems, vol. 11, no. 4, pp. 447-455, 1975.
[9] E. H. Shin, "Estimation techniques for low-cost inertial navigation," UCGE report, 2005.

[10] E. H. Shin and N. El-Sheimy, "Accuracy improvement of low cost INS/GPS for land applications," Department of Geomatics Engineering, University of Calgary, 2001.

[11] J. E. Bortz, "A new mathematical formulation for strapdown inertial navigation," IEEE Transactions on Aerospace and Electronic Systems, vol. 7, no. 1, pp. 61-66, 1971.

[12] M. Park, "Error analysis and stochastic modeling of MEMS based inertial sensors for land vehicle navigation applications," Department of Geomatics Engineering, University of Calgary, 2004.

[13] Specification of AHRS400CC, 2012, http://www.xbow.com.

[14] P. S. Maybeck, Stochastic Models, Estimation, and Control, vol. 1, Academic Press, New York, NY, USA, 1979.

[15] X. Niu, "A proposed evaluation standard for the navigation results of MEMS INS/GPS integrated systems," in Proceedings of the International Symposium on GPS/GNSS, Taipei, Taiwan, 2010.

[16] IMU-FSAS, 2012, http://www.novatel.com/assets/Documents/ Papers/FSAS.pdf.

[17] MTi-G, 2012, http://www.xsens.com/images/stories/products/ PDF_Brochures/mti-g\%20leaflet.pdf. 


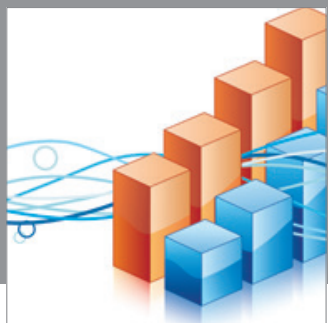

Advances in

Operations Research

mansans

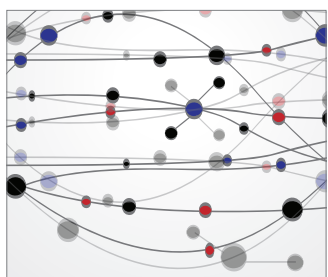

The Scientific World Journal
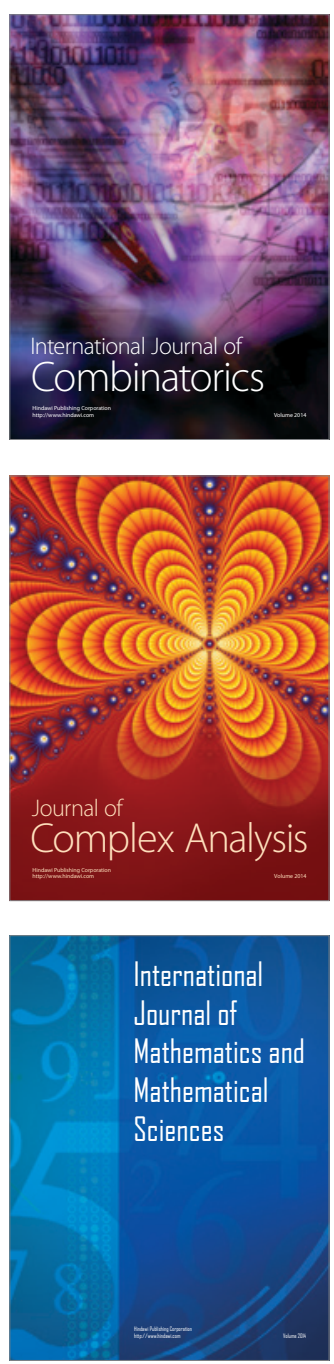
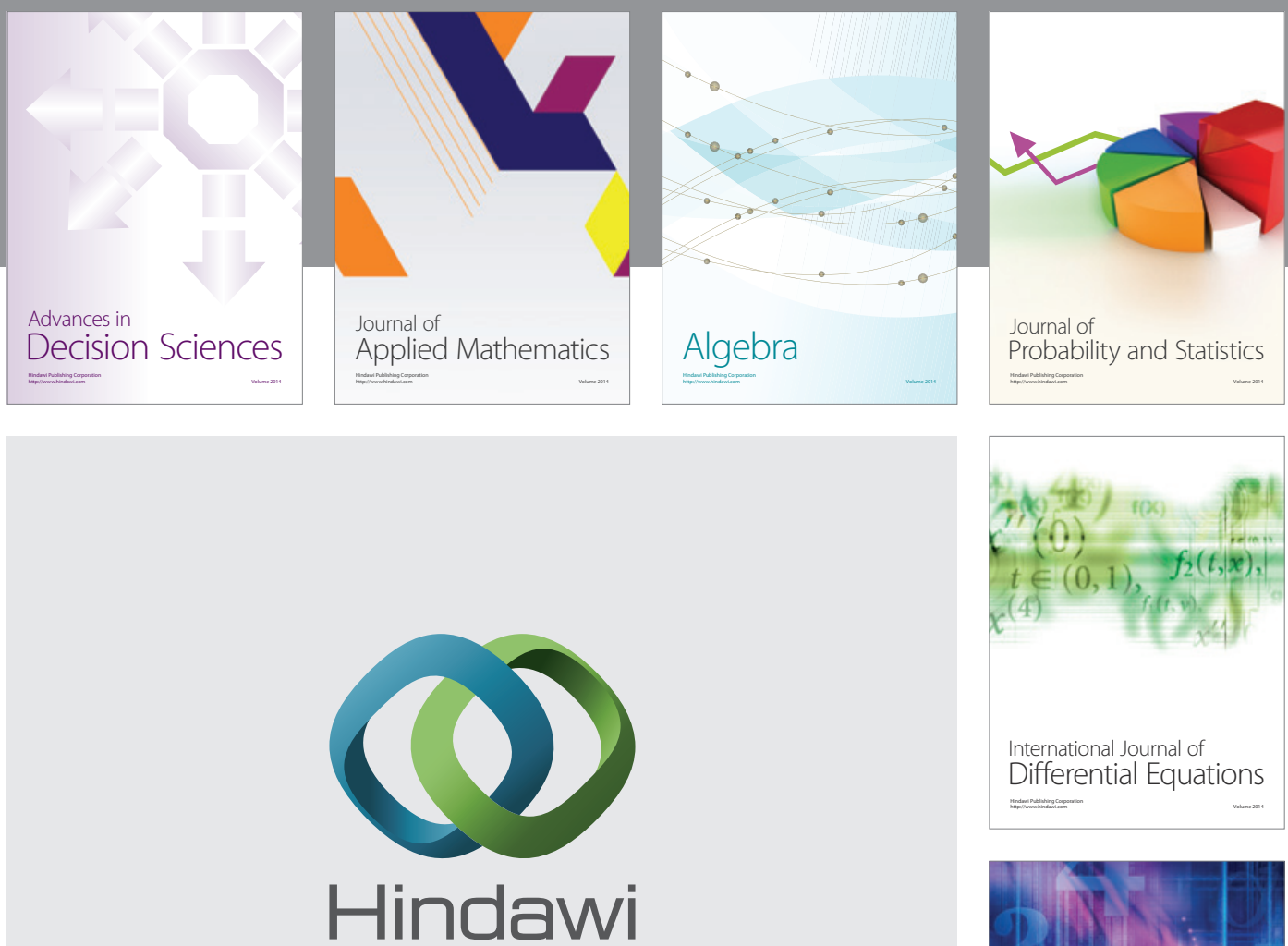

Submit your manuscripts at http://www.hindawi.com
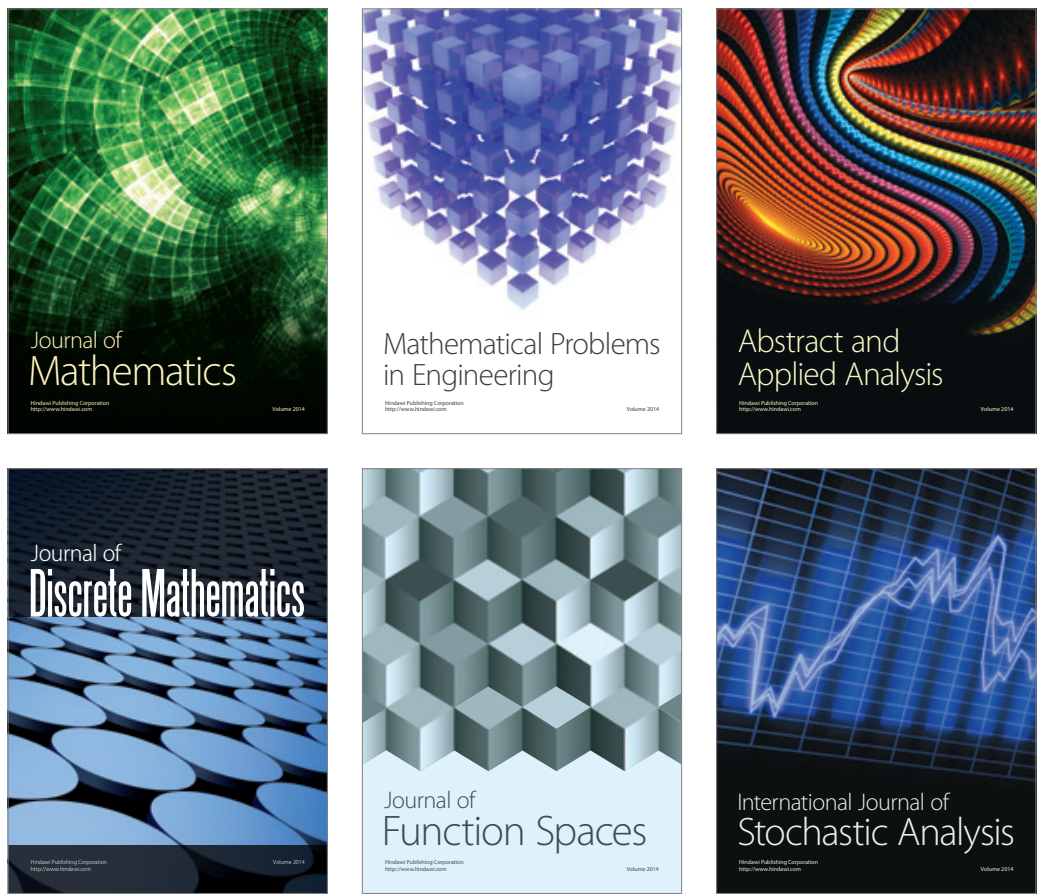

Journal of

Function Spaces

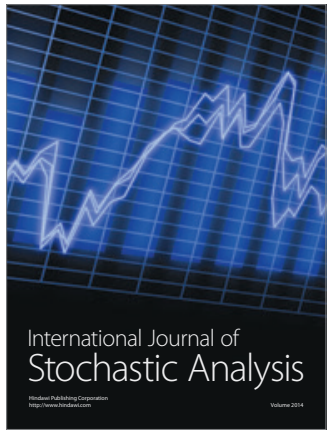

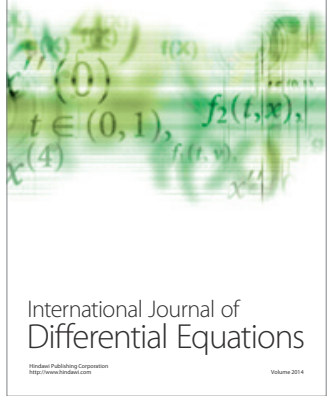
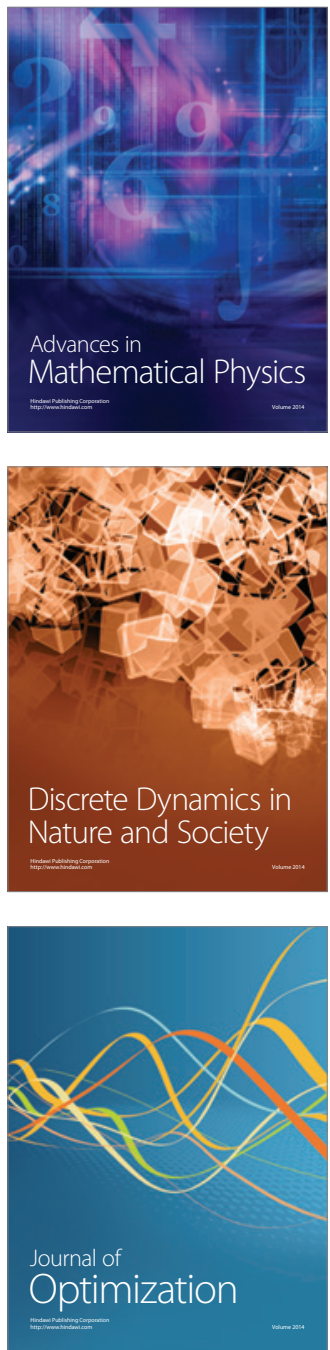